\title{
Biomaterials II: Corrosion Issues in Biological Environments
}

\author{
Vilupanur A. Ravi
}

The longevity of bioimplants is dependent on the nature of their interactions with the physiological environment in which they are placed. For metallic implants, insights into in vivo durability can be obtained using in vitro test methods with the caveat that the latter are only an approximation of the final environment that the component(s) will encounter. In particular, electrochemical techniques afford relatively quick and efficient pathways to obtain information relative to the environmental stability of candidate alloys. Four papers addressing different aspects of the corrosion of metallic implants follow this commentary. They are briefly discussed in the sequence in which they appear in the following pages.

Douglas Hansen discusses the interaction of biomolecules with metallic surfaces in his paper titled "Biological Interactions at Metal Surfaces." The author traces the formation of biofilms by commencing with Quincke's electrical double layer, going through the DLVO theory, and ends by discussing the specificity of binding sites via the "lock and key" mechanism. He also describes the forces and interactions involved in biomolecule adsorption (i.e., hydrogen bonding, ionic or electrostatic interactions, and charge transfer interactions). He then discusses the interaction between biomolecules and an oxide surface. The passivating oxide layer on the surface of Type 304L stainless steel is discussed in the context of complexation by a protein.

Nicholas Kirkland et al. discuss in vitro corrosion and associated mechan- ical performance of $\mathrm{MgCaZn}$ alloys. The authors report on the corrosion behavior and mechanical compression properties for a series of $\mathrm{MgCa}, \mathrm{MgZn}$ and $\mathrm{MgCaZn}$ alloys. The compression strengths for these different alloys after immersion times of 1 and 3 weeks in simulated body fluid and also for alloys that were not immersed at all are presented. The authors discuss the corrosion mechanisms in relation to the effects of alloying with calcium and/ or zinc. They emphasize that alloy selection based on mechanical properties before corrosion should be avoided. The authors also report on an artificial neural network incorporating both corrosion current densities and yield strengths with the goal of being a better predictor of performance. They conclude that by modifying the $\mathrm{Zn} / \mathrm{Ca}$ ratio a suitable balance between strength and dissolution rates can be attained.

In the next paper, I.M. Pohrelyuk et al. explore the corrosion resistance of nitrogen surface-treated Ti-6Al-4V alloys in $0.9 \mathrm{wt} . \% \mathrm{NaCl}$. They report on the influence of nitriding temperature and pressure on the resultant microstructure of the titanium alloys and their subsequent corrosion resistance. The authors point out how process conditions can affect the relative amounts of the coating constituents (i.e., titanium nitrides $\left(\mathrm{TiN}\right.$ and $\left.\mathrm{Ti}_{2} \mathrm{~N}\right)$ and a diffusion layer of nitrogen in titanium) in the final microstructure. They also report on their potentiodynamic studies of these surface-modified alloys and control samples. The authors discuss their results and the role of the microstructural constituents on the surface-modified alloys and the effects of processing that led to the microstructural changes in the first place.

Finally, Vilupanur Ravi et al. report on the corrosion behavior of a new class of boron-containing titanium alloys in physiologically relevant media in their research summary. They also discuss the suitability of these alloys from a mechanical perspective along with implications for alloy design. The effects of thermomechanical treatments of the alloys on the corrosion behavior are also discussed. The authors report results from cyclic potentiodynamic polarization tests conducted in deaerated phosphate buffered solutions at $37^{\circ} \mathrm{C}$ and in 0.9 wt. $\% \mathrm{NaCl}$. The authors conclude that the addition of $\mathrm{B}$ to pure titanium offers a promising route to achieve increases in strength with modest to nil increases in stiffness while maintaining the excellent corrosion resistance of pure Ti. They support this conclusion by pointing out that the addition of up to $0.4 \mathrm{wt} . \% \mathrm{~B}$ to pure $\mathrm{Ti}$ results in an increase of $\sim 128 \%$ in the yield strength while causing an increase of only $\sim 4.6 \%$ in the stiffness.

In summary, this selection of papers highlighting different aspects of metallic implants provides a snapshot of the different areas of active research in the field of corrosion in biological environments.

Vilupanur Ravi is Professor and Chair in the Department of Chemical and Materials Engineering at California State Polytechnic University, Pomona and is the advisor to JOM from the Corrosion and Environmental Effects Committee of the Structural Materials Division of TMS.

\section{Vilupanur A. Ravi is a TMS Member!}

To read more about him, turn to page 10. To join TMS, visit www.tms.org/Society/Membership.aspx. 